\title{
No todo es tromboembolismo: sarcoma intimal de arterias pulmonares
}

\section{It is not All Thromboembolism: Intimal Sarcoma of the Pulmonary Arteries}

\author{
J. Iglesias Gordo ${ }^{1}$ M.J. Viso Soriano ${ }^{2}$ \\ D. Villa Viñas ${ }^{1}$ \\ L. Ferrando Lamana² \\ A. Apestegui Garmendia 3
}

${ }^{1}$ Servicio de Radiología, Hospital Universitario Miguel Servet,
Zaragoza, España
2 Servicio de Anatomía Patológica, Hospital Universitario Miguel
Servet, Zaragoza, España
${ }^{3}$ Servicio de Urgencias, Hospital Universitario Miguel Servet,
Zaragoza, España

Rev Argent Radiol 2019;83:26-29.

Estimados Editores,

El sarcoma intimal de arterias pulmonares es una patología muy poco frecuente que suele confundirse inicialmente con el tromboembolismo pulmonar (TEP) mediante hallazgos por tomografía computarizada (TC), en un determinado contexto clínico. En ambas entidades, los pacientes presentan una presentación similar, fundamentalmente con disnea, dolor torácico y tos, por lo que al identificar un defecto de repleción en las arterias pulmonares en la TC se puede atribuir de forma errónea al TEP. ${ }^{1}$ El manejo terapéutico es completamente distinto y sólo un diagnóstico temprano puede aumentar la esperanza de vida de los pacientes.

Presentamos el caso de una mujer de 72 años con antecedentes de carcinoma ductal infiltrante de mama en remisión seis años antes, que acudió a urgencias por dolor torácico, disnea y tos seca persistente. Tenía una angiografía coronaria reciente normal y los análisis de laboratorio revelaron como valor destacable un dímero-D de $742 \mu \mathrm{g} / \mathrm{l}$. Con esos datos se solicitó desde el servicio de urgencias una angio-TC de arterias pulmonares (-Fig. 1) donde se apreciaba un voluminoso defecto de repleción en la arteria pulmonar derecha que se diagnosticó como TEP. La paciente fue anticoagulada y controlada con mejoría inicial, pero cuatro meses después volvió a urgencias con empeoramiento de la disnea, tos y hemoptisis. En las radiografías de tórax (-Fig. 2) se apreciaba un aumento de tamaño de arterias pulmonares con una masa parahiliar izquierda y atelectasia completa de lóbulo superior izquierdo (LSI), mejor valorados en la angio-TC torácica (-Fig. 3), donde persistía la ocupa-

received

December 14, 2017

accepted

March 18, 2018

published online

July 13, 2018
Address for correspondence J. Iglesias Gordo, MD, Servicio de Radiología, Hospital Universitario Miguel Servet, Zaragoza, España (e-mail: jdiglesias@salud.aragon.es).

ción arterial con unas características que, unidas a su evolución, sugirieron un origen tumoral. Se realizó biopsia a través de broncoscopia de la masa que obstruía el bronquio del lóbulo superior izquierdo, con un resultado histológico de sarcoma intimal de arterias pulmonares (-Fig. 4), patología muy poco frecuente y de difícil diagnóstico diferencial con el TEP.

El sarcoma intimal de las arterias pulmonares fue descrito por primera vez por Malndelstamm en 1923. Se cree que se origina en células pluripotenciales de la íntima, de ahí su denominación. Se caracteriza por crecimiento intimal con obstrucción de la luz, siendo tumores mesenquimales pobremente diferenciados con rasgos fibroblásticos o miofibroblásticos. ${ }^{2}$ Los marcadores endoteliales son negativos y de forma ocasional puede observarse diferenciación heteróloga (rabdo u osteosarcomatosa). Se objetiva expresión nuclear inmunohistoquímica de mouse double minute 2 homolog (MDM2) en el 70\% como resultado de la amplificación de la región 12q 12-15 que contiene el proto-oncogén MDM2,3 el cual puede promover la formación de tumores actuando sobre el supresor tumoral p53.

Se trata de una entidad muy rara, de etiología desconocida con pocos casos descritos, que suele diagnosticarse en torno a los 50 años sin una clara predilección de sexo. ${ }^{3}$ Frecuentemente hay un retraso en el diagnóstico debido a que tanto la clínica como los hallazgos en TC son similares a los encontrados en el tromboembolismo pulmonar, ${ }^{4}$ principal patología con la que se suele confundir. Los síntomas son superponibles, siendo los más frecuentes disnea progresiva,
Copyright @ 2019, Sociedad Argentina de Radiología. Publicado por Thieme Revinter Publicações Ltda., Rio de Janeiro, Brazil. Todos los derechos reservados.
License terms

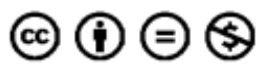

10.1055/s-0038-1642628. ISSN 1852-9992. 


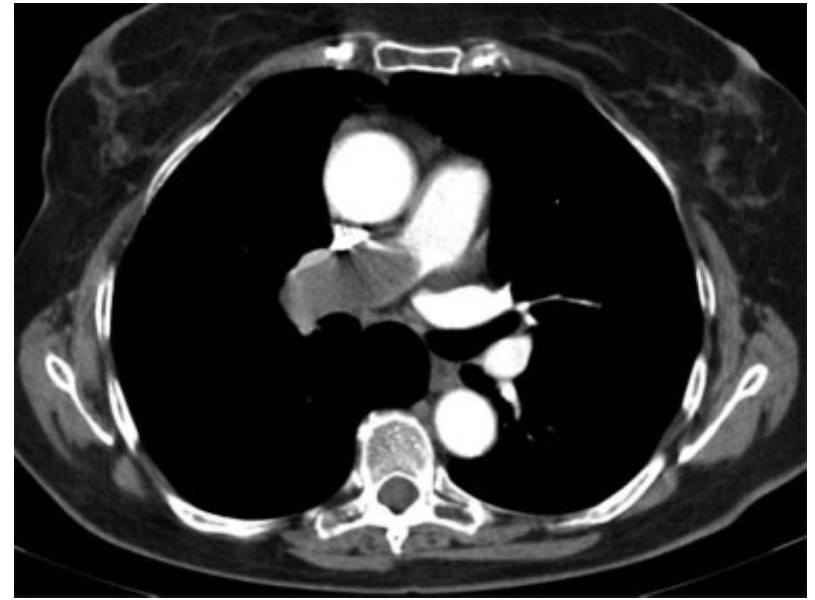

Fig. 1 Angio-tomografía computada (TC) de arterias pulmonares donde se observa un voluminoso defecto de repleción que ocupa totalmente la luz de la arteria principal derecha con incremento del calibre del vaso. En el contexto clínico propuesto se sugirió el diagnóstico de tromboembolismo pulmonar.

tos y dolor torácico. En caso de encontrar un curso progresivo en lugar de súbito, junto a una clínica constitucional con fiebre, fatiga y pérdida de peso, así como elevación de marcadores inflamatorios en suero como velocidad de segmentación globular (VSG), proteína C reactiva (PCR) y lactato deshidrogenasa (LDH), pueden ayudar a orientar el cuadro de una forma correcta. ${ }^{5}$ Asimismo, la ausencia de factores de riesgo para la enfermedad tromboembólica, o bien una falta de respuesta clínica y radiológica a la terapia anticoagulante en caso de diagnóstico erróneo inicial de TEP con empeora-

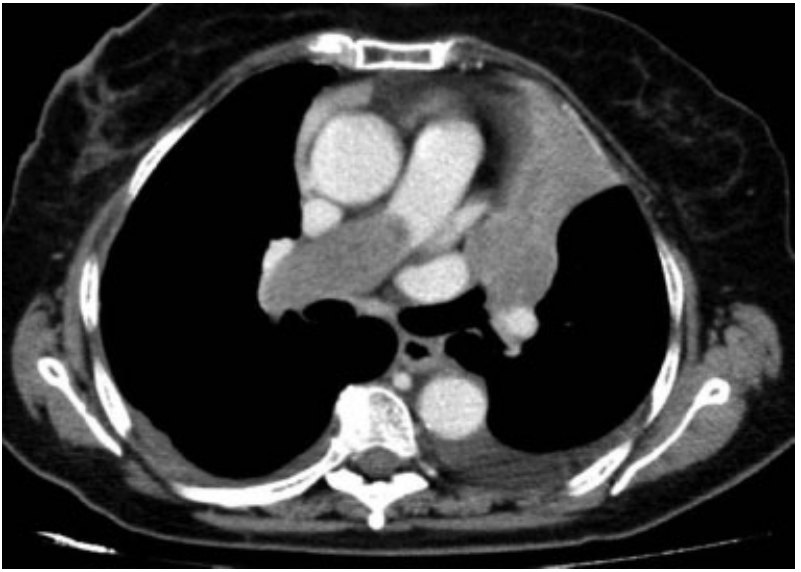

Fig. 3 TC torácica con contraste con ventana de mediastino donde se observa la persistencia de la ocupación de la arteria pulmonar derecha, con leve crecimiento respecto a la TC previa y con cierto grado de realce tras la administración del contraste yodado, por lo que se sugirió un origen tumoral. Además, se observa la masa que obstruye totalmente el bronquio del lóbulo superior izquierdo produciendo atelectasia completa, con la misma atenuación y realce que la masa que ocupa la arteria. Hay leve derrame pleural bilateral tal y como se apreciaba en la radiografía.

miento progresivo debe elevar la sospecha para hacer un análisis minucioso.

En la radiología simple el sarcoma de arterias pulmonares puede presentarse con hallazgos inespecíficos como ensanchamiento de arterias pulmonares, hiliar o mediastínico, así como con cardiomegalia, nódulos pulmonares o disminución de la vascularización pulmonar. ${ }^{6}$ La prueba de imagen más usada que ayuda a enfocar el cuadro es la TC

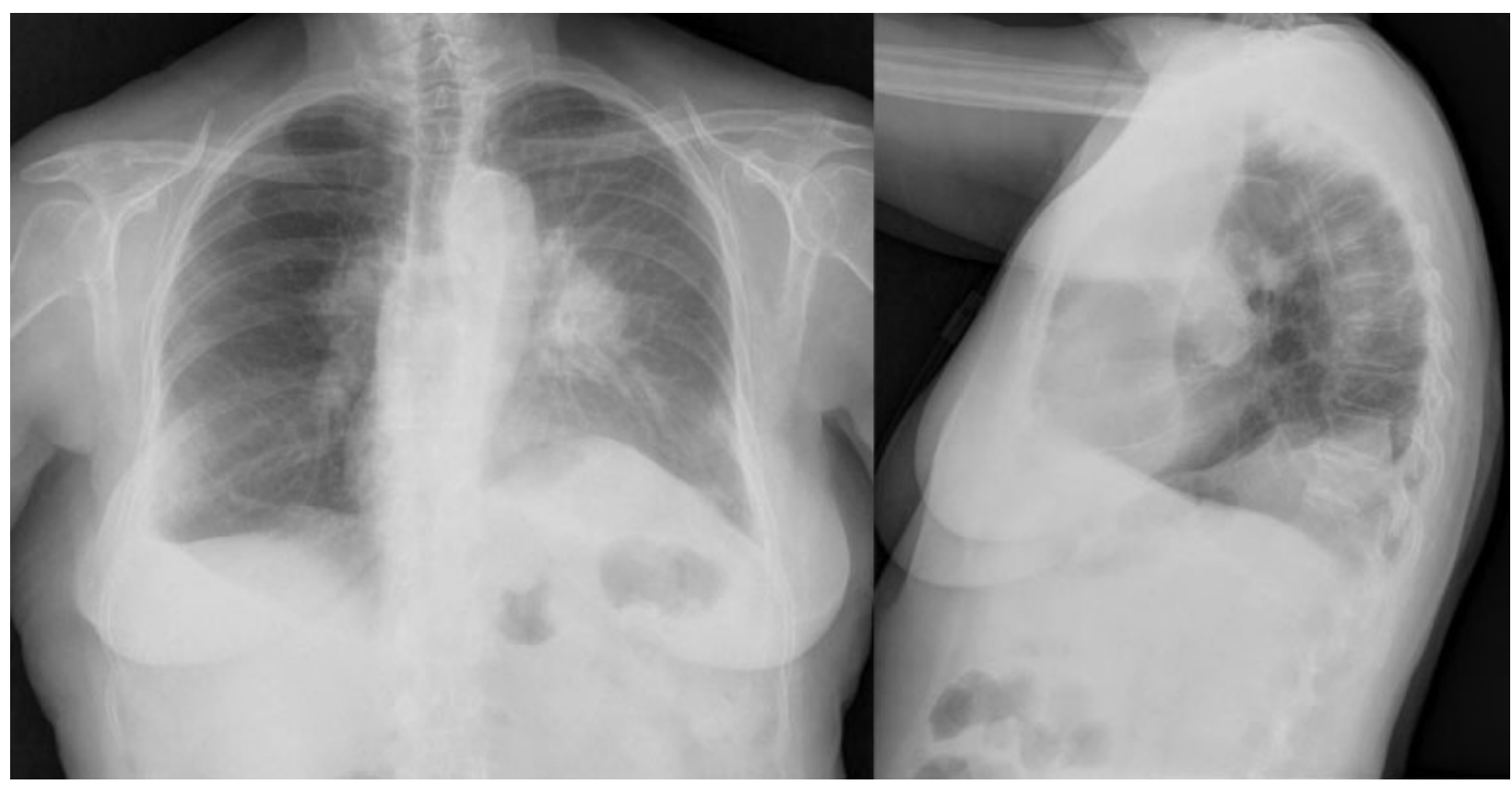

Fig. 2 Radiografías posteroanterior (izquierda) y lateral de tórax (derecha) en las que se aprecia un aumento de tamaño de las arterias pulmonares y una masa parahiliar izquierda que sugiere ser la causa del colapso completo de lóbulo superior izquierdo, demostrado por la pérdida de volumen, el desplazamiento de la cisura mayor, del bronquio principal izquierdo y la elevación del hemidiafragma así como por el aumento de la atenuación respecto al lado contralateral. Hay leve derrame pleural bilateral asociado. 


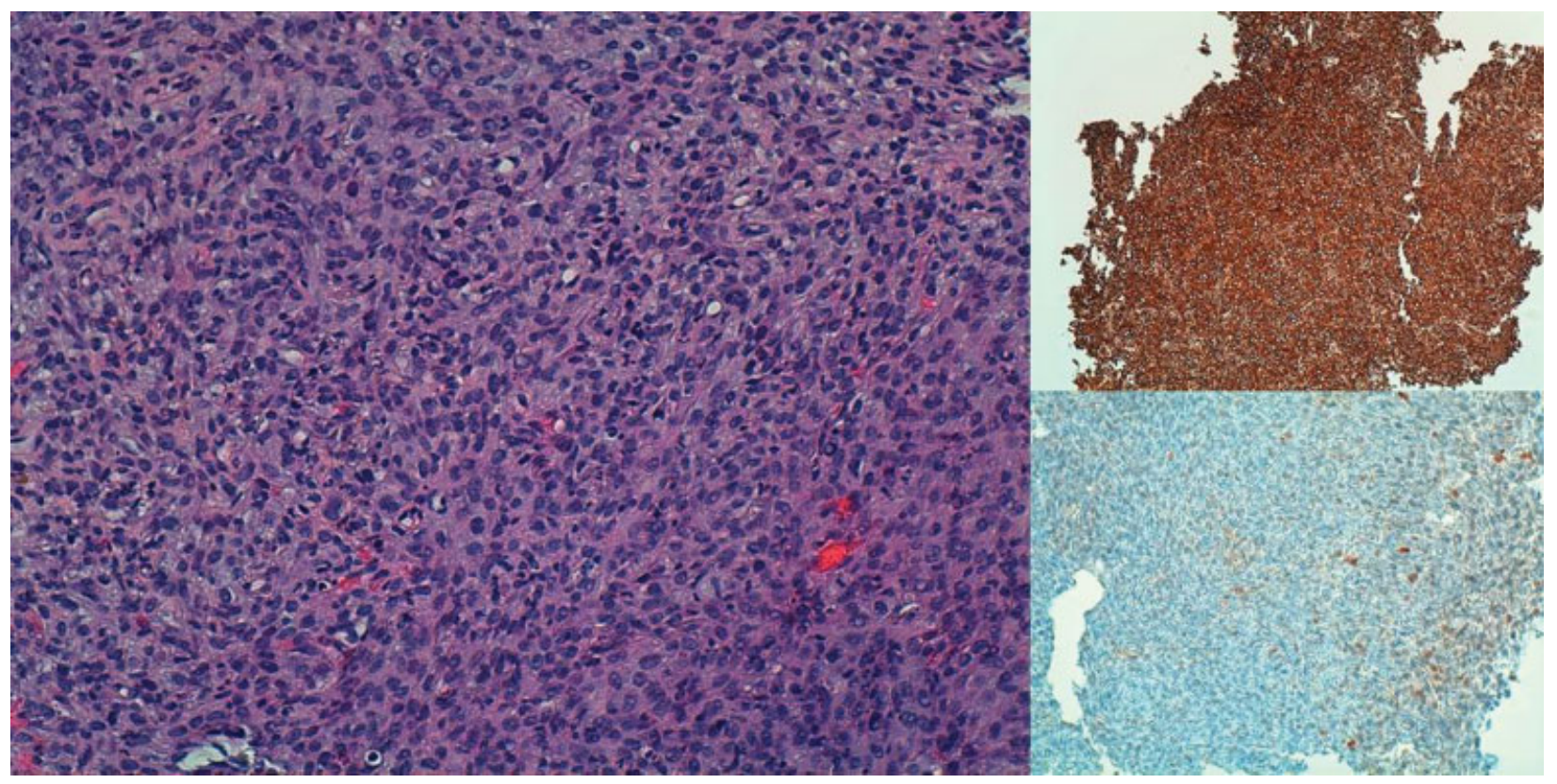

Fig. 4 En la parte izquierda de la imagen (tinción con hematoxilina-eosina $\times 20$ ) se observa una proliferación fusocelular densa con atipia moderada. En la parte superior derecha (tinción con vimentina $\times 10$ ) se aprecia que expresa de forma intensa vimentina, característica típica de los sarcomas. Abajo a la derecha mouse double minute 2 homolog 40 (MDM2), se objetiva sobreexpresión nuclear de MDM2, hallazgo característico del sarcoma intimal.

con contraste, donde el signo más frecuente es la identificación de un voluminoso defecto de repleción con baja atenuación situado en el tronco y/o en las arterias pulmonares principales. Los datos que pueden orientar a malignidad son: ensanchamiento del calibre del vaso, presencia de realce tras la administración de contraste y, en casos evolucionados como hallazgo ya distintivo, la extensión extraluminal o metastásica fundamentalmente a pulmón o mediastino. ${ }^{7}$ Por lo tanto, se debe prestar mucha atención si el paciente tiene una clínica leve y manifestaciones por imagen altamente sospechosas, especialmente si el dímeroD está en rango normal, en esos casos hay que orientar el proceso como maligno. Ante la sospecha tumoral puede recurrirse a otras técnicas de imagen como la resonancia magnética, donde son hallazgos característicos la detección de perfusión y la presencia de realce ${ }^{8}$ o bien a la tomografía por emisión de positrones (PET) TC, en la que un aumento de la captación de ${ }^{18} \mathrm{~F}$-FDG con un $\operatorname{SUV}_{\text {máx }}$ elevado ${ }^{9}$ confirmarían la necesidad de biopsia ante la alta probabilidad de origen neoplásico.

Generalmente el pronóstico es malo debido a la alta agresividad del tumor, con una esperanza de vida en general inferior a 18 meses. El tratamiento quirúrgico representa la única opción curativa $\mathrm{y}$, en caso de poder realizarse, las posibilidades incluyen la endarderectomía pulmonar, la lobectomía o bien la neumonectomía, según el grado de afectación. ${ }^{10}$ Suele acompañarse de quimio y radioterapia adyuvantes o incluso neoadyuvantes, aunque son necesarios más estudios para demostrar su utilidad. En casos evolucionados se realiza tratamiento paliativo.

\section{Responsabilidades Éticas}

Protección de personas y animales. Los autores declaran que para esta investigación no se han realizado experimentos en seres humanos ni en animales.

Confidencialidad de los datos. Los autores declaran que han seguido los protocolos de su centro de trabajo sobre la publicación de datos de pacientes.

Derecho a la privacidad y consentimiento informado. Los autores declaran que en este artículo no aparecen datos de pacientes.

\section{Confidencialidad de los Datos}

Los autores declaran que han seguido los protocolos de su centro de trabajo sobre la publicación de datos de pacientes y que todos los pacientes incluidos en el estudio han recibido información suficiente y han dado su consentimiento informado por escrito.

Conflicto de Intereses

Los autores declaran no tener ningún conflicto de intereses.

\section{Bibliografía}

1 Ebner L, Huber A, Ott D, Christe A. Pulmonary intimal sarcoma: a rare differential diagnosis for arterial filling defects on a chest CT. Acta Radiol Short Rep 2014;3(02):2047981613514052

2 Huo L, Moran CA, Fuller GN, Gladish G, Suster S. Pulmonary artery sarcoma: a clinicopathologic and immunohistochemical study of 12 cases. Am J Clin Pathol 2006;125(03):419-424

3 Fletcher C, Bridge J, Hogendoorn P, Mertens F. WHO classification of Tumours of Soft Tissue and Bone: pathology and genetics of tumours of soft tissue and bone. 4th ed. Lyon: IARC Press; 2013 
4 Bendel EC, Maleszewski JJ, Araoz PA. Imaging sarcomas of the great vessels and heart. Semin Ultrasound CT MR 2011;32(05):377-404

$5 \mathrm{Pu}$ X, Song M, Huang X, et al. Clinical and radiological features of pulmonary artery sarcoma: A report of nine cases. Clin Respir J 2017. Forthcoming

6 Restrepo CS, Betancourt SL, Martinez-Jimenez S, Gutierrez FR. Tumors of the pulmonary artery and veins. Semin Ultrasound CT MR 2012;33(06):580-590

7 Singla Long S, Johnson PT, Hruban RH, Fishman EK. CT features of pulmonary artery sarcoma: critical aid to a challenging diagnosis. Emerg Radiol 2010;17(02):153-155
8 Schuler PK, Weber A, Bode PK, et al. MRI of intimal sarcoma of the pulmonary arteries. Circ Cardiovasc Imaging 2009;2(05): e37-e39

9 Lee EJ, Moon SH, Choi JY, et al. Usefulness of fluorodeoxyglucose positron emission tomography in malignancy of pulmonary artery mimicking pulmonary embolism. ANZ J Surg 2013;83 (05):342-347

10 Wong HH, Gounaris I, McCormack A, et al. Presentation and management of pulmonary artery sarcoma. Clin Sarcoma Res 2015;5(01):3 